\title{
Genomic Epidemiology of Cardiovascular Disease, Adoption of the Health Belief Model to Increase Screening for Known Risk Factors and Use of Natural Approaches to Enhance Heart Health
}

William Ebomoyi $E^{*}$

Department of Health Studies College of Health Sciences, Chicago State University, Chicago, Illinois, USA

\begin{abstract}
This project reviewed genomic epidemiology of cardiovascular diseases, identified specific gene-chromosomes which are linked to the onset of various heart problems. The economic burden associated with heart diseases was estimated to be well over $\$ 475.3$ billion as of 2009. Innovative genomic science has revealed the need to educate the American and global community about the relevance of adopting the family history approach combined with the utilization of the health belief model to screen for the various risk factors associated with the leading causes of death not only in United States but in many other developed nations. The investigator presented the role of innovative medical diagnostic resources used in the management of cardiovascular diseases in United States. As a primary preventive strategy, the inexpensive natural pathway to heart heath was provided.
\end{abstract}

Keywords: Genomic epidemiology; Cardiovascular disease; Family history activities; Advanced medical dagnostic equipment; The health belief model; International hapmap consortium; University of chicago Medicine center for care and discovery; Natural pathways to heart health

\section{Genomic Epidemiology}

Mausner and Bahn defined epidemiology as "the scientific study of the determinants and distribution of diseases and injuries in human population" [1]. In the same vein, lilienfeld and Stolley conceptualized epidemiology as the study focusing on the patterns of diseases occurrence in human populations and the factors that influence theses patterns [2]. Khoury Muin and associates further explained genetic epidemiology as "the study of the role of genetic factors and the interaction with environmental factors in the occurrence of diseases in populations [3]. In the age of genomic science, genomics implies the interaction of genes with other genes and the environment. Genomebased innovative technologies have revolutionized scientific knowledge about the incipient onset of various cardiovascular diseases.

Genomics has completely revolutionized our hitherto understanding about the various precursors for complex diseases such as the CVD and associated risk factors which were previously unknown due to the egress of technological science. In fact, the accomplishment of the human genome sequencing, bioinformatics and advanced, computerized analytic techniques continue to reveal numerous unknown gene-chromosomes which illuminate the underlying reasons why certain individuals are more at risk for cardiovascular disease than their hardier counterparts.

In this eclectic review of pub Med, and Google, for advanced scientific literature search, we refer to cardiovascular diseases as the full spectrum of diseases which include stroke, or cerebrovascular diseases. The main diseases of the circulatory system include acute rheumatic fever, chronic rheumatic heart diseases, hypertensive diseases, ischemic heart disease, pulmonary heart diseases, and those diseases of pulmonary system, cerebrovascular diseases, diseases of arteries and capillaries, diseases of veins, lymphatic vessels, and lymph nodes, and unspecified disorders of the circulatory system.

Cardiovascular diseases did not become the leading cause of death in United States until 1940. Before then, pneumonia was the most lethal cause of death in United States. By 1940, cardiovascular diseases eventually became the leading cause of death not only in United States but also in many other developed nations [4].

One landmark study which revolutionized our understanding of CVD in the twenty-first century involved the Framingham heart study. This project which was initiated in 1948 was to investigate the epidemic of coronary disease in the United States and successfully characterize the risk factors associated with this deadly disease. In 1948, the Framingham Heart study, under the direction of the present National Heart, Lung and Blood Institute (NHLBI), embarked on a very expensive and ambitious epidemiological project which changed our understanding of cardiovascular health problems. Since 1971, this prominent investigation is now conducted in collaboration with the Boston University. As confirmed by Kannel, the study utilized the prospective epidemiological design, and insights were provided about prevalence, incidence, full clinical spectrum in terms of attrition rates, and the predisposing factors [5]. The recognized risk factors, then which were linked with coronary disease in United States were stroke, peripheral artery diseases and heart failure. The research team dispelled clinical misconception about isolated systolic hypertension, left ventricular hypertrophy, dyslipidemia, atrial fibrillation and glucose intolerance. In view of the economic, physical and emotional distress associated with CVD, this study was designed to:

- Explore the economic burden associated with chronic cardiovascular diseases

*Corresponding author: William Ebomoyi E, Professor, Department of Health Studies College of Health Sciences Chicago State University, Chicago, Illinois 60628-1598, USA, E-mail: eebomoyi@csu.edu

Received July 31, 2013; Accepted September 16, 2013; Published September 23, 2013

Citation: William Ebomoyi E (2013) Genomic Epidemiology of Cardiovascular Disease, Adoption of the Health Belief Model to Increase Screening for Known Risk Factors and Use of Natural Approaches to Enhance Heart Health. J Cardiovasc Dis Diagn 1: 127. doi:10.4172/2329-9517.1000127

Copyright: (c) 2013 William Ebomoyi E. This is an open-access article distributed under the terms of the Creative Commons Attribution License, which permits unrestricted use, distribution, and reproduction in any medium, provided the original author and source are credited. 
Citation: William Ebomoyi E (2013) Genomic Epidemiology of Cardiovascular Disease, Adoption of the Health Belief Model to Increase Screening for Known Risk Factors and Use of Natural Approaches to Enhance Heart Health. J Cardiovasc Dis Diagn 1: 127. doi:10.4172/23299517.1000127

Page 2 of 7

- Discuss the gene chromosomes associated with these complex diseases

- Accentuate the relevance of Family History Approach (FHA) in the identification of intra-familial risk factors underlying onset of cardiovascular disease

- Discuss the relevance of the Health Belief Model in sensitizing at risk groups to participate in primary preventive programs

- Compare the technological and medical diagnostic management of CVD in the developed and developing nations and

- Specify the natural pathways and approaches to heart health

\section{Economic Burden Associated with Chronic Cardiovascular Diseases}

Research studies have revealed that cardiovascular disease and stroke are among the most commonly reported, highly expensive health problems confronting the United States today. Cardiovascular diseases including heart disease and stroke are the first and third leading causes of death and disabilities among both male and female population in United States. In fact, these diseases account for more than 151,000 Americans who died of cardiovascular diseases in 2005. These mortality data account only for the American cohort of 65 and under. Data from the Federal Centers for Disease Control and Prevention(CDC) have indicated that heart disease and stroke are also among the leading causes of disability in the United States workforce with almost I million individuals who are disabled or made paraplegic by stroke alone [6]. Epidemiological projections pinpoint how American life-expectancy would have increased by nearly 7 years if cardiovascular diseases and stroke could be eliminated. In fact, innovative genomics science continues to reveal specific inkling about achieving major breakthrough in combating these insidious diseases.

The cost of managing heart disease and stroke in United States which includes health care expenditure and lost productivity from the mortality and morbidity was estimated to be over 475 billion in 2009 .

Reports from the American Heart Association indicate the cost of management of heart disease and stroke was $\$ 475.3$ billion [7]. This amount includes the direct and indirect cost. In their analysis, direct cost consist of the cost of physicians and other professionals, hospitals and nursing home services, cost of medication, home health care and other medical durable equipment while the indirect cost consists of lost productivity which includes the morbidity and mortality from the diseases. Figure 1 illustrates the most recent trend in the estimated direct and indirect cost of major cardiovascular diseases in United States by 2010 .

By 2010, the total cost of cardiovascular diseases in United States was estimated to be well over $\$ 444$ billion. The treatment of this diseases account for about $\$ 1$ of every $\$ 6$ dollar spent on health care in United States. Due to innovative medical intervention, the overall mortality rate for heart disease and stroke continues to decline; however, rate for incidence and mortality are on the on the rise especially among many minority ethnic groups, particularly those that are economically impoverished [7]. The improvement in the management of these cardiovascular diseases and stroke can be attributed to the monument impact of the accomplishment of the Human Genome Sequencing initiatives and the development pharmacogenomics in the treatment and management of various cardiovascular diseases and stroke and other primary preventive behaviors which include exercise, effective diet, and other behavioral interventions.

\section{Gene chromosomes associated with these complex diseases}

From the haplotype map of human genome, the international HapMap Consortium has cautioned, molecular research scientists, by advising them to realize that despite the ever-accelerating pace of biomedical research, the root causes of common human diseases remain largely unknown, therefore, preventive measures are generally inadequate, and available treatment are seldom curative. The international research team therefore recommended the adoption of family history as one of the strongest risk factors for nearly all diseases, including cardiovascular diseases which serve as the leading cause of death in most developed nations. Prevention research scientists and molecular biologists must, therefore, be poised to use their intuitive knowledge of innovative genomic science to identify the causal genes and variants as this could represent an important step in the path toward meaningful preventive strategies, diagnosis and treatment of most complex diseases [7]. Inherited genes as precursors for heart disease are not modifiable $[8,9]$.

Frazier et al. [10] delineated some of the non-modifiable etiologies of $\mathrm{CHF}$ from a review of genetic studies. These heart failure-associated diseases consist of primary cardiomyopathies, mixed cardiomyopathies that involve altered cardiac muscle including generalized multi-organ disorders and other associated diseases. At present, the Genome Wide Association study has only revealed some inkling about specific chromosomes and specific alleles which are associated with CVD. However, there are over 1400 genes associated with 1200 Mendelian traits and only about 10 to 15 of them are the etiologic genetic variants for complex diseases. Identification of gene-chromosome that code for various cardiovascular diseases is "work in progress", therefore the incessant funding by government, non-profit organizations and biotechnological companies can usher in medical scientific

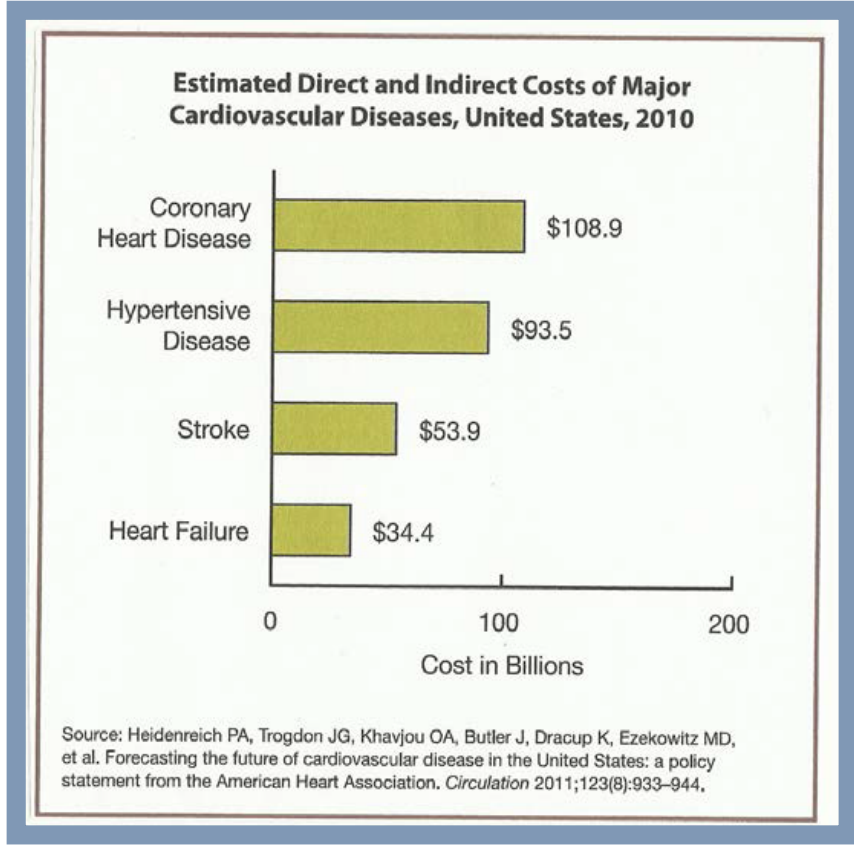

Figure 1: Estimated Direct and Indirect cost of Major Cardiovascular Diseases, United States, 2010 
Citation: William Ebomoyi E (2013) Genomic Epidemiology of Cardiovascular Disease, Adoption of the Health Belief Model to Increase Screening for Known Risk Factors and Use of Natural Approaches to Enhance Heart Health. J Cardiovasc Dis Diagn 1: 127. doi:10.4172/23299517.1000127

Page 3 of 7

breakthrough that could significantly reduce the lethal impact of CVD in the developed and developing nations.

\section{Family History Approach in Identifying Risk Factors for Cardiovascular Diseases}

For many years, families worldwide are usually aware of those single gene and complex diseases which are commonly observed within the immediate and extended family network. Recently, the Federal Centers for Disease Control and Prevention (CDC) provided cogent rationales for the adoption of family history techniques to promptly identify the existing disease burden which are most prevalent in each family [11]. CDC provided the necessary and sufficient conditions which include family members sharing genes, behaviors, lifestyles, environment and other haplotype characteristics [11]. These variables cumulatively influence their health status and their risk of chronic and degenerative diseases such as coronary heart disease, diabetes, and other complex diseases. CDC emphasized specifically, individuals who have a close family member with chronic diseases may have an increased risk of developing similar diseases than those without such intrafamilial linkage [11]. Our understanding of the concept of pedigree is inextricably linked to the benefits derived from family history in the primary prevention of many complex diseases. Genetically, pedigree is the illustration of a family tree. The pedigree is applied by geneticists, modern genome epidemiologists and other medical professionals to evaluate families and attempt to identify patterns or indications which may be helpful in detecting a patient's health status (Figure 2).
The U.S Surgeon General's Family history Initiative is the brainchild of Dr. Richard H. Carmona. By December, 2003 he had advised the National Children Study Federal Advisory Committee in Atlanta GA, to use the week of Thanksgiving when family traditionally gather to compile those genetic and other health problems which are common within the family. By November 2005, Dr. Carmona, the former surgeon general advanced this initiative by launching the new and easier to use web-based instrument entitled: "My Family Health Portrait." Recent observations by the investigator at the Hilton Hotel in Chicago, Illinois have revealed how many families across United States use any national holiday such as the July 4 th commemoration, and the Labor Day in September for this intra-familial gathering. Among those health-professionals within these relatives, it will be very productive to insert family history activity as a component of their agenda.

As illustrated in Figure 2, family history is a documented graphical record of disease frequencies and the observed genetic and complex diseases which are quite common within the immediate and extended family members. Pertinent family health historical record involves illustration of at least three generations of biological relatives. Valid demographic and epidemiological data will include the time age of diagnosis, and the age of onset of specific disease. The etiological agents of the specific disease such as age, gender, and exposure to stress are recorded. Family history is a relevant tool for understanding health risks and prevention of diseases (Figure 2) (Table 1).

In gathering family history, with a focus on screening for risk factors

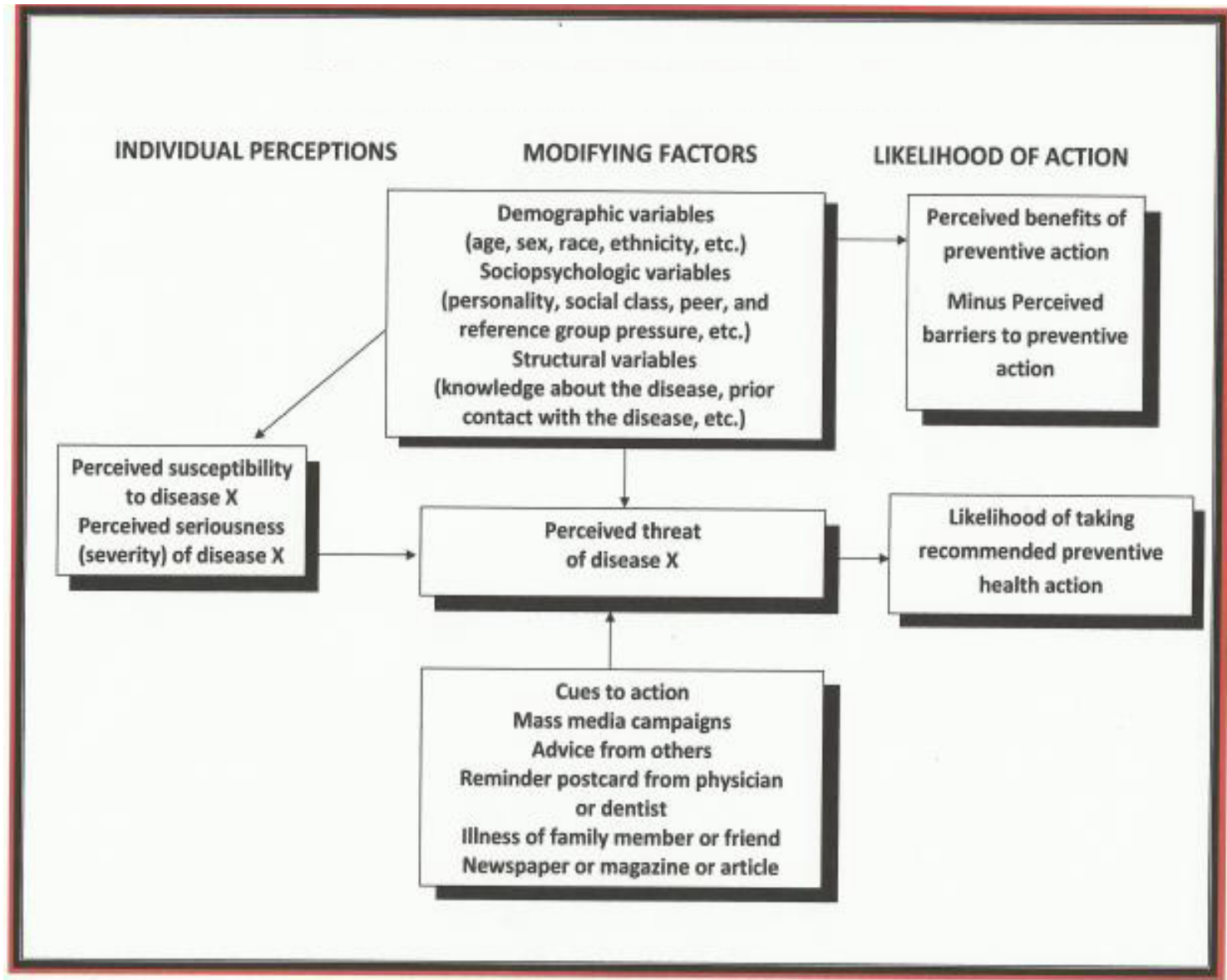

Figure 2: The Health Belief Model (Hochbaum, 1958) 
Citation: William Ebomoyi E (2013) Genomic Epidemiology of Cardiovascular Disease, Adoption of the Health Belief Model to Increase Screening for Known Risk Factors and Use of Natural Approaches to Enhance Heart Health. J Cardiovasc Dis Diagn 1: 127. doi:10.4172/23299517.1000127

Page 4 of 7

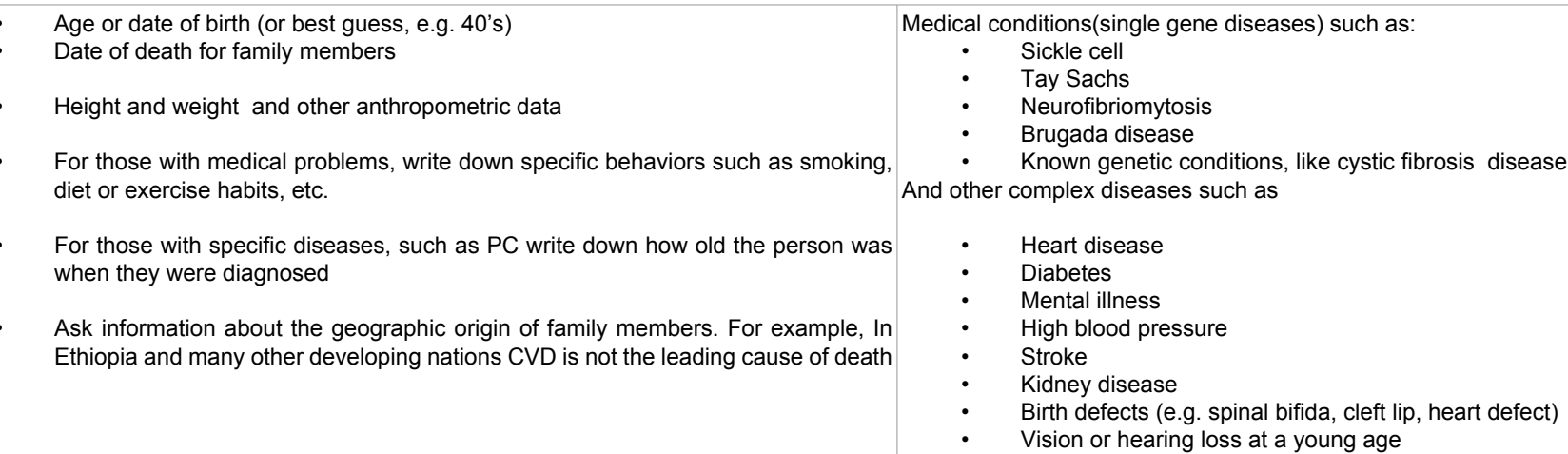

Table 1: Typical family history record will include the following: These data can be used to prevent and manage the onset of CVD.

for CVD, the investigators must document a comprehensive list of all elderly family members including those that are deceased. Efforts must be invested to remember the elderly patriarch. Elderly grandparents are listed and they are asked to provide names of their parents and their grandparents and their great grandparents. Attention is paid to eliciting the names and types of diseases that they suffered from, which became the underlying cause of death. The other components of human pedigree include the brothers, the sisters, including halfsiblings, uncles, aunts, cousins, nephews and nieces.

\section{Adoption of the Health Belief Model to disseminate Global Health Education}

Modern genome epidemiologists and prevention research scientists routinely assess logically and statistically, the theoretical models which are most applicable in explaining individual health behaviors that could maximize public health worldwide and minimize the harm from cardiovascular disease worldwide. The most appropriate model for controlling the incidence of CVD is the Health Belief Model (HBM) developed by Geoffrey Hochbaum and associates Rosenstock, in1966 [12]. This theoretical model was developed in the 1950s to explain why medical screening programs offered by the U.S. Public Health Service were not very successful [12]. The original concept focused on how human health behaviors are determined by personal belief or perception about a given disease. To Hochbaum a person's perception is influenced by the range of intrapersonal perceptions affecting a given behavior [12]. That perception which depends on personal factors involves the level of education, and the parental background of the individual. The person's phenomenological experience about the agonizing knowledge that one's parents who may have died from CVD or encountered the morbidity associated with CVD, and eventually died from the disease can trigger psychological outburst of painful memories. This fact is the underlying knowledge about perceived susceptibility of CVD.

The major theoretical constructs are illustrated in Figure 3. The key components of the HBM consist of perceived susceptibility to a disease such as heart disease. Genomic interventions and the application of the magnetic resonance imaging have revealed the genetic association between gene-chromosomes and the various cardiovascular diseases. The associated etiological agents for CVD are family history, age and high cholesterol levels among other risk factors.

\section{Perceived seriousness of cardiovascular diseases}

Scientific data reported by World Health Organization indicated that CVD will continue to serve as one of the leading causes of death even in the developing nations by 2020. Today, the American Heart
Association indicts CVD as the current leading cause of death in United States (Figure 1).

\section{Modifying variables}

The major modifying variables identified are the demographic characteristics, socio-psychological and structural variables. These variables include age gender, income and educational background of the individual. Those who are older than 40 are more at risk of suffering from CVD than teenagers. The less educated members of society engage more frequently in smoking, consumption of food items that are rich in lipids than the highly educated members of society. The non-modifiable variables are inherited gene-Chromosomes for complex disease, age, and other anthropometric indicators and other haplotype characteristics.

\section{The perceived threat of Cardiovascular Diseases}

Professor Geoffrey Hochbaum meticulously suggested that health behavior is influenced by cues to action [12]. To him, cues to action, are specific events, people, innovative discoveries that motivate people to change their behaviors. Today, innovative genomic epidemiology consistently reveals several lethal variants of gene-chromosomes for myocardial infarction.

\section{Among the various cues to action}

The most powerful motivating cue is the death of one's father, mother or brother more so, when a younger sibling is having high blood pressure and low blood pressure. An international team of researchers has successfully identified new gene variants associated with an increased risk of Myocardial Infarction (MI). The new genes they identified were detected after having studied a million genetic biomarkers in $1,200 \mathrm{MI}$ patients $[11,13,14]$.

Apart from post cards mailed to clients by their family physicians, there is a high frequency of public health service announcements mass media campaigns designed to promote public heart health. Health education programs are provided in many churches whereby volunteer health professional teach the congregation members about the availability of inexpensive screening services for high blood pressure, cholesterol screening and stress test, being performed in nearby clinics (Figure 3).

\section{Likelihood of action}

Using this framework, the likelihood of taking action, such as screening CVD could be quite high. On reflecting on commonsense and scientific theory, Hochbaum maintained that scientists need to be 
Citation: William Ebomoyi E (2013) Genomic Epidemiology of Cardiovascular Disease, Adoption of the Health Belief Model to Increase Screening for Known Risk Factors and Use of Natural Approaches to Enhance Heart Health. J Cardiovasc Dis Diagn 1: 127. doi:10.4172/23299517.1000127

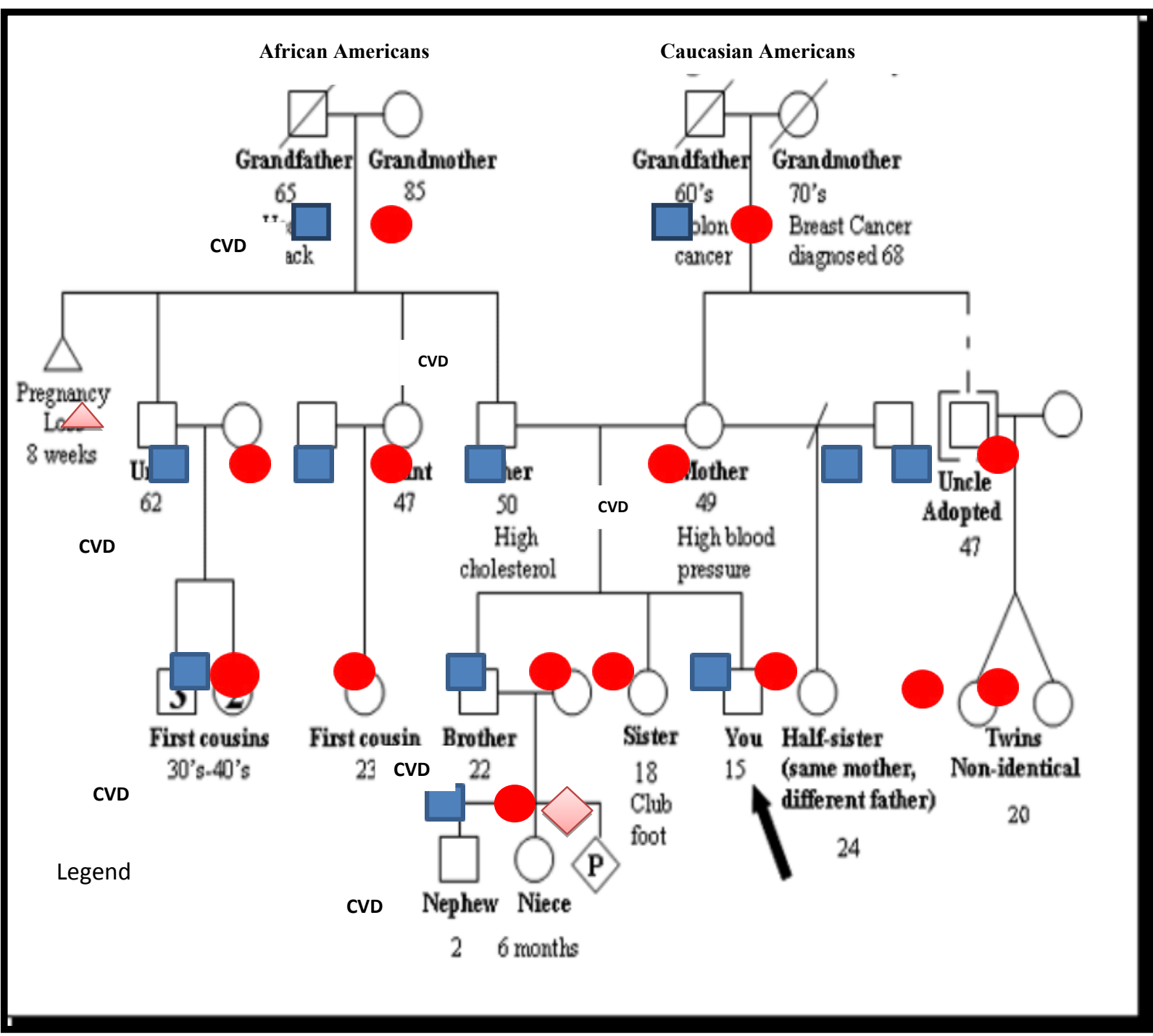

There are scientifically accepted

determination of family history

Cardiovascular Disease

Figure 3: Pedigree from the National Society of Genetic Counselors website: www.nsgc.org.

mindful about Kurt Lewin's widely cited pronouncement that "there is nothing as practical as a good theory $[15,16]$.

\section{Self-efficacy}

When the HBM was conceptualized in the 1950s, the concept of self-efficacy was not a component of the model. A plethora of studies by several socio-psychologists created the impetus to incorporate selfefficacy. Dr. Bandura [17] integrated self-efficacy which he defined as the conviction that one can successfully execute the behavior required to produce the outcome. It is also described as one's ability to perform a certain task or specific function. Self efficacy which Bandura [17] later described as Social Cognitive Theory (SCT), explained that behavior is determined by expectancies and incentives:

(a)Expectancies about environmental cues that is, the belief about how events are connected

(b)Expectancies about the consequences of one's own actions (that is, opinions about how individual behavior is likely to influence outcomes) which is described as outcome expectations and

(c) Expectancies about one's own competence to perform the behavior needed to influence outcomes

Based on Bandura's views, incentive (or reinforcement) is defined as the value of a particular object or outcome. The outcome in this discourse, is preventing the deadly consequences of breast cancer In personal communication with Bandura, he passionately echoed how the development of self-efficacy in children, who are at risk cohort is developed through parental nurturing, instilling confidence and a sense of self-worth in one's children to enable them to develop based on their genetic endowment [17].

\section{Perceived Benefits of preventive action}

The likelihood of taking the desired action is contingent on the perceived benefits of preventive action. Regarding CVD the awareness 
Citation: William Ebomoyi E (2013) Genomic Epidemiology of Cardiovascular Disease, Adoption of the Health Belief Model to Increase Screening for Known Risk Factors and Use of Natural Approaches to Enhance Heart Health. J Cardiovasc Dis Diagn 1: 127. doi:10.4172/23299517.1000127

Page 6 of 7

of preventing mortality and morbidity associated with these diseases outweighs the multiple barriers to taking the relevant action such as primary prevention which includes weekly blood pressure screening. This process provides a relatively simple, low cost technique of early detection that can be performed more frequently than the comprehensive clinical screening shown using the expensive medical devices. The perceived barriers to primary prevention are cost of physician visits for the overwhelming number of women worldwide that are financially indigent. Clinical sites which are not readily accessible to the public and the increased cost of bypass surgeries, MRI screening which could be in the range of $\$ 30,000-\$ 40,000$ and this financial hardship is very common in many parts of the wor ld.

\section{Technology and Medical Diagnostic Management of CVD}

The escalating cost of medical management of cardiovascular diseases in the developed nations is due principally to the use of sophisticated technological equipment to perform the diagnosis of various cardiovascular diseases through non-invasive devices. Besides, the precursors for hitherto life-threatening heart diseases are now better understood and more efficiently managed by the team effort of a team of physicians, nurses and other paramedical personnel.

The non-invasive medical equipment includes electrocardiogram, nuclear stress test (EKG), Echocardiogram (ECHO), Positron Emission Tomography (PET) and computer tomography and Magnetic Resonance Imaging (MRI). The most recently developed non-invasive medical technologies include the following:

\section{Computed tomography (CT)}

CT scan is the test which combines instantaneous $\mathrm{x}$-ray scanning with multiple computed tomographies to reveal detailed images of the heart arteries without surgery. Pictures of the heart are taken by rotating a camera called detector around human body. The patient lies on a specially designed narrow bed which is comfortably moved through the camera's area of focus.

\section{Nuclear cardiology stress test}

Nuclear Cardiology stress test is a test applied as a component of medical diagnosis to measure the heart's ability to respond to external stress in a controlled clinical environment. The stress response is commonly induced by exercise instead of drug stimulation. Cardiac stress tests compare the coronary circulation while the patient is at rest with the same patient's circulation observed during maximum physical exertion. Quite often the physician will inject a radioactive substance into the blood of CVD patient and use gamma x-ray camera to visualize the movement of blood through the heart. This non-invasive procedure is able to detect the movement of blood through the heart. The test can reveal how the heart is functioning in keeping itself saturated with oxygen rich blood; this test is conducted twice to confirm cardiac performance at rest and under severe stress.

\section{Cardiovascular magnetic resonance imaging (Cardiovascular MRI)}

This medical technique utilizes powerful magnets to create a field that sets the nuclei of atoms in heart cell vibrating. The oscillating atoms emit radio signals which are converted by computer into either stationary or moving 3-D images. This noninvasive procedure which visualizes the heart and the vascular structures and functions without exposing patients to radiation and iodinated contrast dye is widely used across the nation.

\section{Echocardiogram}

Echocardiogram also called Trans-Thoracic Echocardiogram (TTE) Harmless ultrasound waves which are quite similar to the ones used in taking sonograms of a fetus are aimed at the chest and bounces off the heart's walls and valves. A computer performs analysis of these rebounding waves and precisely calculates the size and movement of the structure of the heart. Again, physicians usually perform two echogram of the heart, one at rest and the other under stress.

\section{Electrocardiogram (EKG)}

This medical device simply scans and records the electrical impulses which regulate one's heart's pumping action. When there are deviations from normal rhythm pattern; such irregularities are symptomatic of the probability of damaged heart tissue and impeded blood flow.

\section{Positron emission tomography (PET) and CT}

This non-invasive procedure provides structural and functional information about the heart in one scanning bout. Physicians use $\mathrm{CT}$ to locate specific narrowed regions along the arteries; and PET is efficiently used to locate portions of the heart muscles that are deprived of blood flow $[18,19]$.

By December 2012, our research team was invited to the Centers for Care and Discovery advances in medicine, at the world-renowned University of Chicago. Many physicians and nurses who were at specific high-tech floors informed us that scientific programs have been developed at the university in cardiac catheterization, minimally invasive and robot-assisted surgery, cardiovascular surgery and heart transplantation that are flawlessly performed. We also meticulously observed interventional suites and operating rooms in the hospital which featured sophisticated diagnostics and leading-edge medical technologies for cardiac care and vascular care including the state-of the science imaging [20].

Our research team attentively sieved the comments from the interventional cardiologists at the University of Chicago Medical Center who echoed "our cardiologists, cardiac surgeon and vascular surgeons work together to ensure patients get the most appropriate care whether medical, interventional or surgical. Our physicians at the University of Chicago medicine are known for their expertise in ventricular assist device implants, heart valve repair and replacement, cardiac and heart and multiple-organ transplants. Our vascular surgeons are pioneers in the treatment of complex diseases of the arteries, including hybrid procedures for abdominal aortic aneurysms, carotid artery disease and peripheral arterial disease [20].

The issues raised by our research team involved how payment is defrayed by the large number of cardiovascular disease patients who are financially impoverished. Besides, what are some of the financial implications of involving an eclectic team of physicians in the management of heart disease by physicians with sundry sub-specialties? Are there many patients who are clinically refused treatment because they do not have viable insurance coverage? Although we did not receive comprehensive and precise responses about these tantalizing issues, we must emphasize that robotic surgery has numerous advantages instead of the life-threatening thoracic surgeries which are known to create lasting scar tissues. 
Citation: William Ebomoyi E (2013) Genomic Epidemiology of Cardiovascular Disease, Adoption of the Health Belief Model to Increase Screening for Known Risk Factors and Use of Natural Approaches to Enhance Heart Health. J Cardiovasc Dis Diagn 1: 127. doi:10.4172/23299517.1000127

Page 7 of 7

\section{Natural Path to Heart Health}

In addition to compliance with one's physician advice, the key pathways to heart health include exercise, diet, meditation and consistent screening to ensure that one is able to detect the incipient stage of cardiovascular diseases when the management of the diseases can be most effective. Physicians and nutritionists have observed that the combined nutrients in the consumption of assorted fruits, vegetables and whole grain which had been analyzed to contain numerous phyto-chemical nutrients cannot be matched by pharmaceutical drug regimens with have painful side-effects.. The identified heart enhancing vegetables include cruciferous vegetables as broccoli, cauliflower, kale, cabbage and Brussels sprout. The leafy vegetables which are also quite protective against CVD consist of lettuce, spinach, Swiss chard, endives, beet greens and romaine.

The umbelliferous vegetables are celery, parsley, fennel, carrots and parsnip. Vegetables which are the alliums are garlic, onions, shallots, chives and leek. The legume series include soybeans, peas, chickpeas, lima beans, peanuts and dried beans and lentils. The cucurbitaceous group of vegetables includes pumpkin, squash cucumber, muskmelon and watermelon.

The most appropriate exercise for cardiovascular health includes walking, bicycling, swimming, aerobic jogging, elliptical machines, dancing and circuit training. Most prevention-oriented physicians recommend at least 30 minutes of exercise at least four days a week. The health promotion initiatives most appropriate for CVD patients and survivors are gardening, shopping and household chores. These physical activities have the potential to improve human self-esteem, physical fitness, body composition and overall quality of life. Finally, engaging in meditation and routine yoga practices are known to temporarily alleviate the onset of atrial fibrillation. Conscientious breathing exercises are also known to reduce the impact of hypertension. To ensure that our personal pathway to health is smooth, it is advisable for us to confide in our physicians and nurses all the newly adopted practices that we periodically engage in so as to enable our health care providers to provide us the most scientific and effective advice about our heart health.

\section{Acknowledgments}

We write to express my gratitude to the staff and management of the University of Chicago Medicine, Center for Care and Discovery, for a very thorough guide through their advanced and ultra-modern medical facility and their professional expertise in educating us about their medical resources and their magnanimity and transparency in allowing us to carefully obtain pictures of their highly sophisticated medical diagnostic equipment. Without the painstaking characteristics of Mr. James $\mathrm{U}$ Ebomoyi, BA, this project would not have been accomplished. His technological expertise has improved the quality of this manuscript. Finally, we are grateful to Stephen Devries, MD, a physician at the Northwest University Medical Center for his lecture series on heart health and the value of exercise to human health.

\section{References}

1. Mausner JS, Bahn AK (1986) Epidemiology: An introductory text, WB Saunders, Philadelphia, 321-330.

2. Lilienfeld DE, Stolley PD (1994) Foundation of epidemiology. (3rdEdn), Oxford university press, New York.

3. Khoury MJ, Bealy TH, Cohen BH (1993) Fundamental of genetic epidemiology Oxford University Press, New York.

4. Pearson TA, Mensah GA, Alexander RW, Anderson JL, Cannon RO 3rd, et al. (2003) Markers of inflammation and cardiovascular disease: application to clinical and public health practice: A statement for healthcare professionals from the Centers for Disease Control and Prevention and the American Heart Association. Circulation 107: 499-511.

5. Kannel WB (2010) Framingham heart study, project of the National heart, lung and blood institute and Boston University.

6. CDC (2013) Report on heart diseases updates.

7. American Heart Association (2011) Circulation 123: 933-941

8. German JB, Roberts MA, Watkins SM (2003) Genomics and metabolomics as markers for the interaction of diet and health: lessons from lipids. J Nutr 133 2078S-2083S

9. Smith JG, Melander O, Lövkvist H, Hedblad B, Engström G, et al. (2009) Common genetic variants on chromosome 9p21 confers risk of ischemic stroke: a large-scale genetic association study. Circ Cardiovasc Genet 2: 159-164.

10. Frazier L, Johnson RL, Sparks E (2005) Genomics and cardiovascular disease. J Nurs Scholarsh 37: 315-321.

11. CDC Family History techniques, 2013

12. CDC Comprehensive Family History Techniques, 2013

13. Strecher V J, Rosenstock IM (1997) The Health Belief Model Handbook of Psychology.

14. McCarthy MI, Abecasis GR, Cardon LR, Goldstein DB, Little J, et al. (2008) Genome-wide association studies for complex traits: consensus, uncertainty and challenges. Nat Rev Genet 9: 356-369.

15. Almasy L, MacCluer J, Wolf PA, D'Agostino RB, O'Donnell CJ (2010) GAW16 Framingham and simulated data.

16. Damani SB, Topol EJ (2009) Molecular genetics of atrial fibrillation. Genome Med 1: 54

17. Bandura A (1977) Social Learning Theory. General learning Press, New York, USA.

18. Tweeten L, Dell K (2005) Healthcare Siemens, GE Time graphic Time 59-71.

19. Futterman LG, Lemberg L (2000) The Framingham Heart Study: a pivotal legacy of the last millennium. Am J Crit Care 9: 147-151.

20. University of Chicago Medicine center for care and discovery www.rvapc com/821-university-of-chicago-medicine-center-for-care- care-and -discovery 